Proc. of the 15th Int. Workshop on Slow Positron Beam Techniques and Applications, Prague, September 2-6, 2019

\title{
Positron Parameters for Atypical Samples
}

\author{
J.M. Urban-Klaehn, ${ }^{a, *}$, K.L. Gering ${ }^{a}$, C.N. TAylor ${ }^{a}$ And C.A. Quarles ${ }^{b}$ \\ ${ }^{a}$ Idaho National Laboratory, P.O. Box 1625, Idaho Falls, ID 83415, USA \\ ${ }^{b}$ Texas Christian University, Dept. of Physics and Astronomy, Box 298840, Fort Worth TX, USA
}

\begin{abstract}
Rocks, minerals, and powdered catalysts, not considered "typical" positron annihilation spectroscopy materials were included in these studies, to find the optimal way to characterize atypical materials by the most appropriate set of parameters in a well-defined environment. The positron annihilation spectroscopies: the Doppler broadening and positron lifetime were compared with other supplementary techniques to validate, interpret, and understand the results in different materials better. It was found that although rocks are heterogeneous mineral aggregates, some positron annihilation spectroscopy values were specific for various types of rocks, potential oil deposits, and their conditions; and quite different for opals due to Ps formation there; moreover in order to characterize properly samples with small mass and density (catalytic powders), their measuring geometry, matrix effect, and set of operational parameters needed to be well-defined.
\end{abstract}

DOI: 10.12693/APhysPolA.137.201

PACS/topics: positron lifetime spectroscopy, Doppler broadening, positronium, rocks

\section{Introduction}

Positron annihilation spectroscopy (PAS) technologies found broad applications in studies of nanoporous materials: polymers, zeolites, metal-organic frameworks (MOFs), as well as for metals with defects. They are also well suited for probing the phase or morphology change due to temperature or stress in well-characterized materials. In this work we investigated unconventional materials with not well-defined and non-homogeneous structures by use of the PAS analysis in attempts to find the best material-specific PAS technology parameters to characterize these materials. Our analysis include rocks, opals, and catalytic powders.

\section{Experimental techniques}

For the Doppler broadening spectroscopy (DBS), high purity Ge detectors with resolution $1.2 \mathrm{keV}$ at $569 \mathrm{keV}$ were used, with the Ge-68 or Na-22 source sandwiched between two sample pieces. For powdered samples at room temperature kapton film with metal backing was used to confine the powder, while for vacuum/temperature measurements a closed stainless container was applied. The DBS background correction was done by the step-like function suggested by Gunnick [1].

The positron annihilation lifetime (PAL) setup was done in fast-fast coincidence system with the Na-22 source and Hamamatsu photomultipliers, with the PAL resolution less than 300 ps. Since these projects spread over several years, multiple software programs were utilized for PAL analysis: originally PATFIT and the LT Kansy program for discrete lifetime analysis, supported occasionally by CONTIN and MATLAB for lifetime distribution.

*corresponding author; e-mail: jagoda.urban-klaehn@inl.gov
The X-ray fluorescence (XRF) analysis was provided by Chevron; the scanning electron microscopy (SEM) analysis on JEOL-JSM-6100 with the energy distribution X-ray (EDX) detector. The residual gas analysis (RGAS) was performed by GMC 064 Balzer, with the temperature controlled feedback system, and the improved vacuum system at $4 \times 10^{-7}$ Torr. The surface and porosity analysis was conducted by use of the Brunauer-EmmettTeller (BET) nitrogen absorption method.

\section{PAS parameters for rocks characterization at room temperature}

Variety of rocks samples, mainly sandstones and carbonates (limestones and dolomites), but also marbles, in different conditions: dry, brine, and water soaked, solid, or powdered were examined and then compared to opals.

It was found that the $S$-parameter and the mean lifetime value, $\tau$, were the most reliable representations for rocks. The mean lifetime value was represented as the weight average of the intensities for three discrete lifetime values.

According to Fig. 1, the trend of $S$-parameter versus mean lifetime values was linear, due to no Ps formation. The carbonates had significantly lower $S$-parameter and mean lifetime values than sandstones. All types of rocks were grouped within their own type, starting with marbles, then dolomites and limestones up along the slope; with opals extending upwards and away from the slope due to the Ps presence.

PAS values depended on rocks' conditions, so that the $S$-parameter values were higher for brine, water, and oil soaked samples than for dry ones. The average "dry" $\langle\tau\rangle$ was $261 \pm 7$ ps (sandstones), $287 \pm 18$ ps for brine- and $301 \pm 27$ ps for water-soaked rocks; while for limestones: $217 \pm 7$ ps for dry, $241 \pm 12$ ps for brine- and $256 \pm 17 \mathrm{ps}$ for water-soaked. For the oil-soaked rocks the lifetime values were about one standard deviation higher than for the brine [2]. 


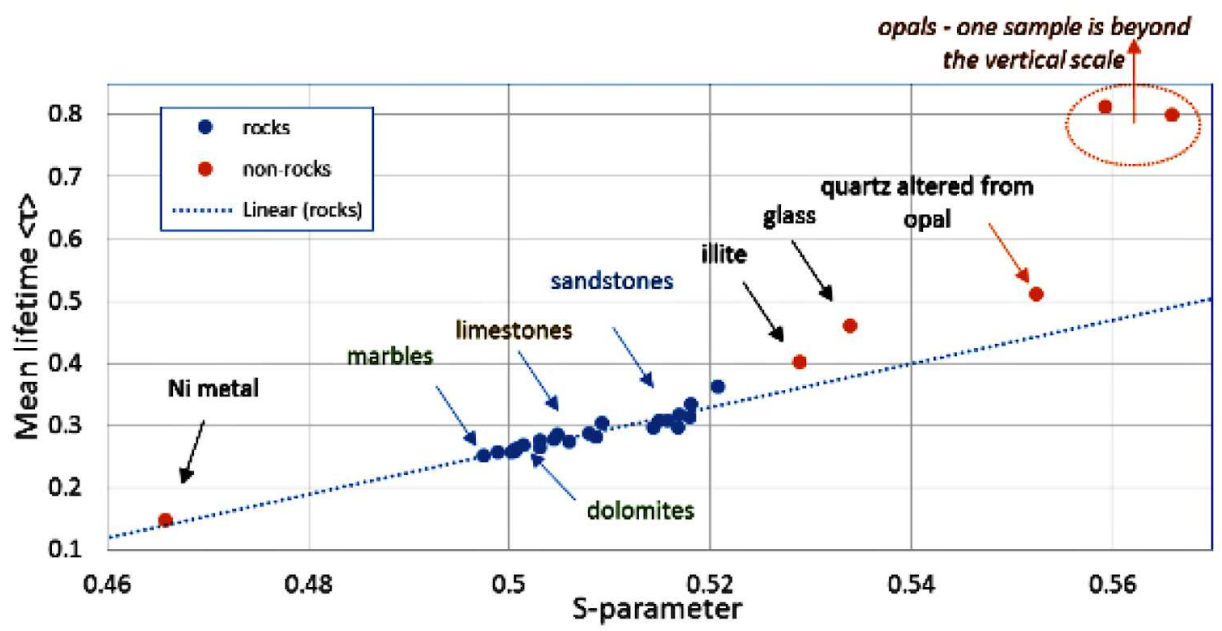

Fig. 1. S-parameter value versus mean lifetime value for rocks, opals and some other samples [2].

The PAS spectroscopies were not only sensitive to the materials structure and sample state but also to the chemical environment. The main difference in PAS values between sandstones and carbonates was not just the total porosity, but also a character of the chemical bond. In sandstone the bond is covalent $\mathrm{SiO}_{2}$, in carbonates it is mainly ionic in $\mathrm{CaCO}_{3}$ between $\mathrm{Ca}^{2+}$ and $\mathrm{CO}_{3}^{2-}$. Welch [3] calculated the so-called Gaussian fraction (GF) which is roughly equivalent with the core penetration for different elements. The larger GF fraction corresponds with smaller $S$-parameter value and the vice versa. Moreover, the core repulsion correlates with the atomic number $Z$ in the following way:

$$
\begin{aligned}
& \mathrm{GF}_{\mathrm{Si}}(=0.08)<\mathrm{GF}_{\mathrm{Ca}}(=0.15)<\mathrm{GF}_{\mathrm{Mg}}(=0.17) \\
& \text { also } S_{\mathrm{Mg}}<S_{\mathrm{Ca}}<S_{\mathrm{Sr}} \text { since } Z_{\mathrm{Mg}}<Z_{\mathrm{Ca}}<Z_{\mathrm{Sr}} .
\end{aligned}
$$

While the core penetration values explained why the $S$-parameter and mean lifetimes values were lower, in general, for carbonates rather than for sandstones, the presence of the admixtures to rocks of the certain type influenced their PAS values within the rock type grouping. For instance within a broad carbonated group a presence of significant amount of magnesium in dolomites and strontium in other carbonates influenced their position within the carbonates as the group (Fig. 1). Magnesium caused more core penetration than calcium, because of less core repulsion due to lower $Z$, so the $S$-parameter value was decreased as a function of magnesium content with the correlation coefficient found experimentally at 0.82. Inversely, the replacement of calcium by heavier strontium caused an increase in $S$-parameter value due to lower core penetration, $r^{2}=0.64[2]$.

\section{The effects of heating to the rock structure; modification of DBS analysis approach}

The DBS values were measured as a function of the temperature. The samples were heated from room temperature up to $220^{\circ} \mathrm{C}$, not to introduce a thermal shock, in a step at $20^{\circ} \mathrm{C} / \mathrm{h}$ up, and at $40^{\circ} \mathrm{C}$ step down. The DBS measurement was performed for about one hour, with at least $0.5 \mathrm{mln}$ counts. The temperature measurements were accompanied by RGAS analysis of the released gases.

It is found that the $S$-parameter values increased almost in all rocks, but the total change did not exceed $1.5 \%$. The increase of the $S$-parameter can be explained mainly by expansion of the crystal volume and increase of lattice vibration, so that the valence electrons are spread over a larger volume of the crystal, and the positron wave function is limited to the interstitial areas.

The PAS of rocks heated up to $220^{\circ} \mathrm{C}$ revealed that the conventional $S$ versus $W$-parameter ranges had to be modified to include the intermediate momentum range which was called $S W$-parameter, to encompass intermediate momentum between $S$ and $W$ ranges. Typically $S$-parameter ranges $510-512 \mathrm{keV}(0-$ $\left.4 \times 10^{-3} m_{0} c\right)$, and $W$ extends below $507.5 \mathrm{keV}$ or above $514.5 \mathrm{keV}\left(13-31 \times 10^{-3} m_{0} c\right)$ until the background level is reached. This division works well in metals with a clear distinction into core versus free electron annihilation effects/regions. However, in rocks the character of the bonds is different. The covalent bonding causes the hybridization of $s p^{3}$ state, thus lowering core penetration, thus extending momentum of bind electron toward lower momentum.

This effect was clearly seen during heating: while the $S$-parameter values were increasing as a function of temperature, both $W$ and $S W$-parameters were decreasing; in some rocks (the Boise sandstone) almost all the change was limited to the $S$ and $S W$-areas and almost none was shown in the $W$-area of high electron momentum annihilation.

Although the $S$-parameter values were lower for limestones than for sandstones at room temperature, the ratio of $S$-parameter change versus temperature was higher for limestones compared to sandstones: in Spergen LS $\Delta S / \Delta T=0.3 \%$ per $100^{\circ} \mathrm{C}$; in Boise SS $0.1 \% / 100^{\circ} \mathrm{C}$. 


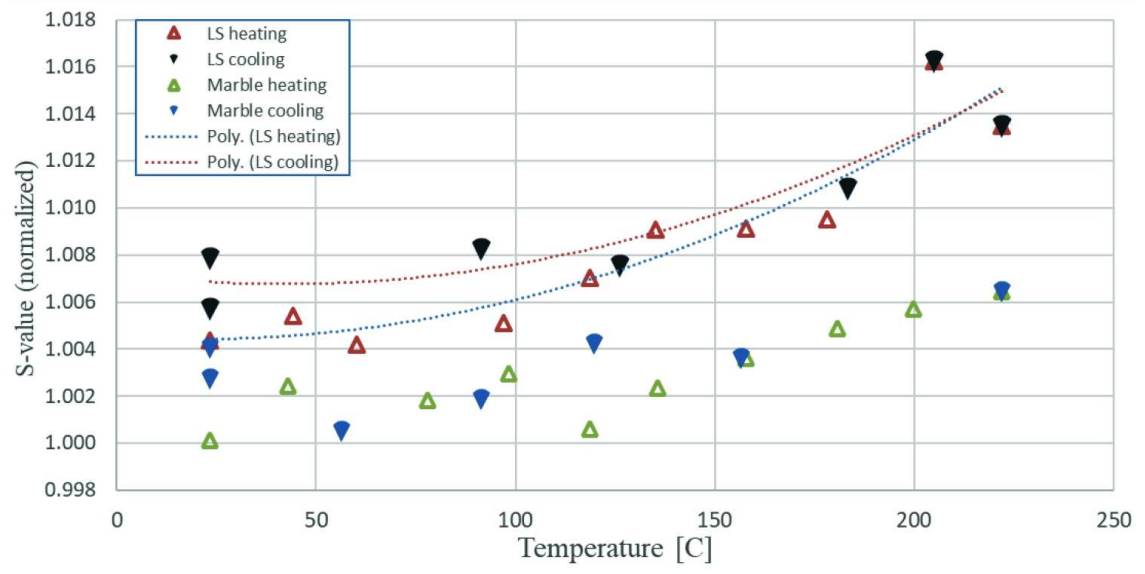

Fig. 2. Normalized $S$-parameter versus temperature for limestones (LS) and marbles (Marb.).

The overall change does not exceed $1.5 \%$ and probably does not cause permanent structure changes. In carbonates, and it was higher for limestones, with the total porosity $10-25 \%$, than for marbles with total porosity not exceeding $0.5 \%$, as expected (Fig. 2).

\section{Opals: origin, diagenesis, and PAS characteristics}

Opals are minerals composed of hydrated silicon dioxide, $\mathrm{SiO}_{2} \cdot n \mathrm{H}_{2} \mathrm{O}$, in a tectosilicate framework structure. They form as deposits of silica shells of organic origin (diatoms, radiolarian, etc.) on ocean floor. Once opal is formed it undergoes transition from amorphous to crystalline with increase in degree of crystallization and the graduate loss of the water. The speed of the process depends on the temperature and $\mathrm{pH}$ conditions of the environment and it goes through the following phases: opal-A (amorphous) $\rightarrow$ opal-CT (cristobalite-tridymite) $\rightarrow$ micro-quartz (quartz/opal) $\rightarrow$ quartz.

The tetrahedral layers of tridymites and cristoballites in the intermediate level of crystallization are characterized by open volume microstructure, with the average void size of $0.52 \mathrm{~nm}$. Water bonds with opal crystals producing indefinite three-dimensional "cages" framework.

Variety of opals of different origin and diagenesis were studied. The samples were characterized by the PAL and DBS measurements and also by the BET and SEM spectroscopies.

Table I shows two longer lifetimes due to Ps formation in opals in different diagenesis stages, compared to some other rocks. There is a natural progression from a carbonate sample and illite (containing some clay and water), through the "quartz/opal" phase to opals in different stages of diagenesis. All opals showed a common lifetime value of about $2 \mathrm{~ns}$, with the intensity at least 10\%; which is higher than $1.8 \mathrm{~ns}$ for Ps formation in bound water, found in illite. Opal CT sample was characterized the longest lifetime at about $33 \mathrm{~ns}$ with intensity $\approx 4.5 \%$, with the same sample analysis done with lifetime distribution allowed, the centroid was shifted to $45 \mathrm{~ns}$, due to
TABLE I

Selected PAL lifetime and intensity values for opals versus other rocks

\begin{tabular}{l|c|c|c|c|c|c}
\hline \hline \multicolumn{1}{c|}{ Sample } & $\tau_{3}$ & $I_{3}$ & $\tau_{4}$ & $I_{4}$ & $\langle\tau\rangle$ & $S$ \\
\hline carbonate & 3.50 & 0.71 & - & - & 0.263 & 0.5007 \\
illite & 1.81 & 1.65 & - & - & 0.402 & 0.5289 \\
quartz/opal & 1.03 & 7.8 & 2.37 & 3.8 & 0.461 & 0.5339 \\
opal A & 1.96 & 10.3 & 16.3 & 1.55 & 0.799 & 0.5659 \\
opal CT & 2.00 & 9.93 & 33.1 & 4.46 & 1.979 & 0.5594 \\
opal W & 2.06 & 16.6 & 13.2 & 1.08 & 0.813 & 0.5593
\end{tabular}

spheroid radii size $12-14 \AA$ [4]. While opal A and opal CT had almost the same $\tau_{3}$ and $I_{3}$ values, their longest lifetimes (Table I) were quite different due to different morphology thus void sizes. The opal-A is a powder with high surface area, its BET hysteresis isotherm analysis shows the shape of the pores with a narrow opening with a wide body impeding oxygen from penetrating to the cavities.

Significant Ps formation caused a deviation from linearity in $S$-parameter versus $\langle\tau\rangle$ which was already described in Fig. 1. The same effect was observed in so called quotient DBS spectrum by comparing different diagenesis stages of opals with the common rocks [5]. The lowest $S$-parameter and thus the highest ratio was obtained for the dolomite, and then there was a natural progression via pure quartz and quartz altered from opal to the opal CT. The "wings" were caused by the difference in the momentum range which was slightly wider for valence electrons annihilation compared to para-Ps momentum free annihilation in opals.

\section{Catalytic powders}

The PAS measurements of zeolite catalytic powders used in catalysis process were carried out to assess microstructure change due to high temperature processing and ageing. The amount of the catalyst material was scarce, with usually less than $0.1 \mathrm{~g}$, and low density, 
since it was obtained from batches of materials processed through chemical reactors of very limited size. Some catalysts were characterized by a very robust internal structure that was not significantly affected by high temperatures. These challenges prompted us to prepare wellcharacterized sample holders to encapsulate powdered samples in thin well-defined kapton films; and also to optimize the geometry and multi-layered analysis to determine well the fraction of positron annihilating in the sample, versus the source, and the matrix; that provided a basis for a multi-layered analysis of kapton as a sensor for the DBS described elsewhere [6].

The initial measurements showed an effect of ageing on so-called "spent" or "cooked" compared to the fresh catalysts. This project was improved by optimizing the process by providing well-defined catalytic pelletized samples with sufficient sample mass, by using larger reactors with uniform conversion rate and well-controlled coking process with few changing parameters.

\section{Summary}

Successful analysis of atypical samples require a targeted set of PAS parameters. After setting the right parameters for specific samples the PAS techniques can be used as a diagnosis tool. The challenges in PAS studies for catalytic powders were instrumental in improving the future approach and applying multi-variant analysis for future experiment in well-defined way, as well as to characterize matrix and geometry effects precisely.

\section{Acknowledgments}

The catalysis work was performed under project 18A12-210FP of the INL LDRD program. The authors want to thank Rebecca Fushimi and her team for catalysis samples and preparation.

\section{References}

[1] K. Debertin, R.G. Helmer, Gamma- and X-Ray Spectrometry with Semiconductor Detectors, NorthHolland, Netherlands 1988, p. 162.

[2] J.M. Urban-Klaehn, C.A. Quarles, J. Appl. Phys. 86, 355 (1999).

[3] D.O. Welch, K.G. Lynn, Phys. Status Solidi B 77, 277 (1976).

[4] T. Goworek, B. Jasińska, J. Wawryszczuk, R. Zaleski, T. Suzuki, Chem. Phys. 280, 295 (2002).

[5] J.M. Urban-Klaehn, C.A. Quarles, "Positron Annihilation in Opals: Evidence of Positronium Formation", 1-56396-825-8/99, AIP, 1999, p. 345.

[6] J. Urban-Klaehn, K.L. Gering, D. Miller, AIP Conf. Proc. 2182, 050019 (2019). 